\title{
Intranasal Analgosedation for Infants in the Neonatal Intensive Care Unit: A Systematic Review
}

\author{
Diane Snyers Sophie Tribolet Vincent Rigo \\ Neonatology Division, CHU de Liège, Liège, Belgium
}

\author{
Keywords \\ Analgosedation $\cdot$ Intranasal route $\cdot$ Infant $\cdot$ Pain \\ management · Preterm infant
}

\begin{abstract}
Aim: Pain management is important for newborns' immediate and long-term well-being. While intranasal analgesia and sedation have been well studied in children, their use could be extended to term and preterm infants. This systematic review aims to assess the use of intranasal medications for procedural analgesia or sedation in the neonatal intensive care unit. Methods: MEDLINE via Ovid, Scopus, Embase, and Cochrane Library were searched independently by two reviewers for clinical studies on sedation or analgesia given intranasally. Results: Seven studies, with 401 patients, were included. The studies described various molecules (midazolam, fentanyl, ketamine, or dexmedetomidine) for different procedures such as intubation in the delivery room, screening for retinopathy, or magnetic resonance imaging. All studies reported significant reduction in pain and sedation markers (based on clinical scales, skin conductance, and clinical variables such as heart rate and crying time). Adverse effects were uncommon and mostly consisted in desaturation, apnoea, hypotension, or paradoxical reactions. Discussion and Conclusion: The intranasal route seems a potential alternative for procedural pain management and sedation in neonates, especially when intravenous access is not avail-
\end{abstract}

able. However, data about safety remain limited. Reported sides effects could be attributed to molecules used rather than the intranasal route. Optimal drugs and doses still need to be characterized. Further studies are needed to ensure safety before promoting a widespread use of intranasal medications in neonatology.

(c) 2022 S. Karger AG, Basel

\section{Introduction}

Infants admitted to the neonatal intensive care unit (NICU) undergo numerous procedures leading to pain and stress during the first days of life [1]. Prevention of pain improves the outcomes and is necessary for ethical reasons. Repeated noxious stimuli early in life expose infants to short- and long-term adverse consequences [2, 3]. In the short term, pain induces behavioural changes, increased stress markers and free radicals, altered cardiovascular parameters, and decreased oxygenation [4]. Recurrent neonatal pain is associated with reduced brain development at term equivalent age [5] and with adverse neurodevelopmental outcomes $[2,6]$.

Pain can be related to disease, but can also arise from procedures and explorations required for intensive care [3]. Therefore, rationalization of care should aim to avoid painful stimuli [1-3]. Each procedure must have a well thought-out indication and needs to be associated with

Correspondence to:

Diane Snyers, diane.snyers@ chuliege.be 
pain assessment and management. Non-pharmacologic strategies have shown variable effectiveness in reducing pain and/or stress-related behavioural responses resulting from mildly to moderately painful or stressful interventions $[2,3]$.

While multiple drugs are available for analgesia and sedation, consensus regarding the ideal molecule is lacking. In neonates, opioids, mainly fentanyl and morphine, are the most common, followed by sedative hypnotics and general anaesthetics $[2,7]$ with considerable variability depending on local practices. Presently, most drugs are administered enterally or intravenously in neonates. Enteral administration may lead to suboptimal analgesia due to a slower onset or a prolonged and unpredictable duration [8]. Intravenous (IV) or intramuscular (IM) administration provides a rapid and effective action, but requires the placement of a catheter or an injection, in itself a stressful and painful procedure [9]. Intranasal (IN) drug delivery has emerged as an alternative method to achieve fast drug delivery while being less invasive [10]. It is used in paediatrics emergency rooms for the administration of analgesics, sedatives, or anticonvulsants $[8,11,12]$, especially when lacking IV access. Several studies also described its use in children for elective procedures including magnetic resonance imaging (MRI), CT-scans, transthoracic ultrasound, electroencephalograms, and hearing tests [13-16].

However, data in neonates regarding doses, pharmacokinetics, or efficacy remain limited. Therefore, uses have derived from paediatric practices. The off-label use of IN drugs for pain and analgesia management in NICU has already been reported in small descriptive studies. Harlos et al. [17] reported IN fentanyl use for palliative care in 58 newborns and small infants, concluding its effectiveness and safety. Kaushal et al. [18] described increasing uses of IN fentanyl in their NICU, most commonly for intubation and central line placement. This systematic review aims to describe the clinical use of IN drugs for analgosedation in the NICU.

\section{Methods}

\section{Research Protocol}

This systematic review was conducted and reported following the Preferred Reporting Items for Systematic Review and MetaAnalysis (PRISMA) Guidelines [19]; the checklist is available in online supplementary data (for all online suppl. material, see www. karger.com/doi/10.1159/000521949). The protocol was registered in advance of data extraction with the International Prospective Register of Systematic Review (PROSPERO) (CRD42020221346).

\section{Eligibility Criteria}

Clinical studies, including randomized controlled trials (RCTs) and prospective or retrospective studies reporting the use of IN drugs for infants' analgosedation in the NICU, were considered eligible. Cohort studies were purposely selected to broaden the data available and to describe current early adopters' practices. Case reports were excluded.

\section{Information Source and Search Strategy}

Two investigators searched MEDLINE via Ovid, Scopus, Embase, and The Cochrane Central Register of Controlled trials for studies published between inception and May 2020, without language restriction. Free language and Mesh/Emtree terms were used, including preterm infant, newborn, analgesia, sedation, pain management, invasive procedure, and IN administration. All search strategies are provided in online supplementary data.

\section{Study Selection}

Citations were managed with the RAYYAN QCRI Web App [20]. After exclusion of duplicates, two reviewers independently screened titles and abstracts for relevant studies. Full texts were then independently reviewed for eligibility. Conflicts at any step of the selection process were resolved by discussion with a third reviewer. Reasons for full-text exclusions are registered in the online supplementary table.

\section{Outcomes}

The primary outcome was the effectiveness of pain management. Given expected heterogeneity in pain assessment, primary outcomes included standardized pain assessment scales and physiologic parameters (heart rate, respiratory rate, oxygen saturation and blood pressure). Secondary outcomes included adverse events, especially cardiorespiratory depression during and after the medication administration, defined by apnoea, oxygen desaturation, bradycardia, hypotension, or chest wall rigidity. Other adverse events like rash, feed intolerance, and paradoxical agitation were also documented. Regional cerebral oxygenation through near-infrared spectroscopy was searched as a surrogate of the impact of medication on cerebral haemodynamic. Additional secondary outcomes included data on pharmacokinetics, optimal dose, and delay before intervention for the different molecules.

\section{Data Extraction and Analysis}

Two reviewers independently extracted pre-specified data using a previously prepared data extraction form. Disagreements were resolved through discussion with a third reviewer. Authors were contacted to provide additional data for missing information.

A large heterogeneity in study designs, sedation indications, drugs used, and pain assessment methods was expected. A descriptive analysis of included studies was performed rather than a metaanalysis.

\section{Bias and Quality Assessment}

Two reviewers independently evaluated risks of bias of individual studies using the revised Cochrane Risk-of-Bias tool for RCT (RoB2) [21]. For prospective and retrospective studies, quality was assessed with the Newcastle-Ottawa Scale (NOS) [22]. 


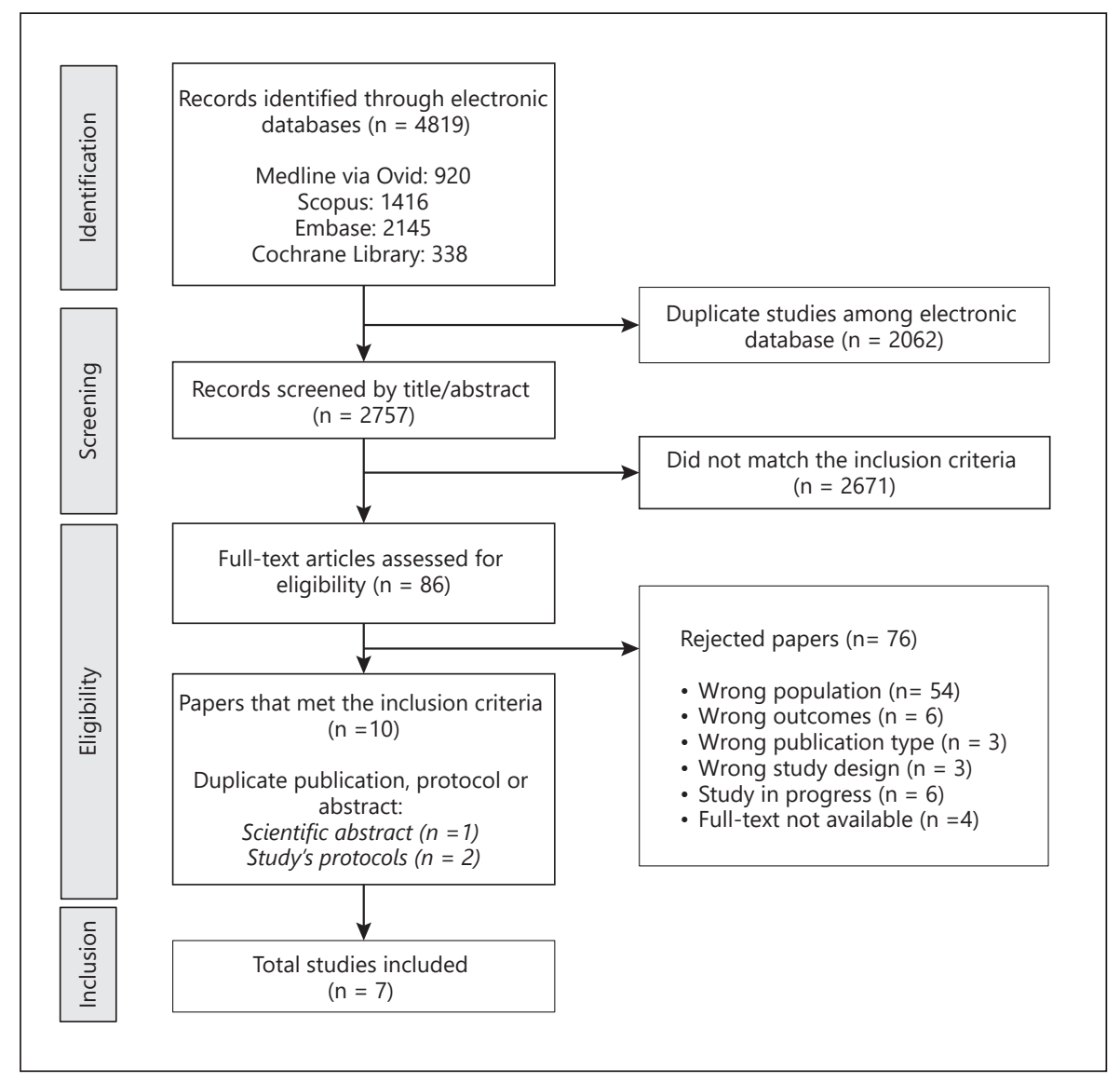

Fig. 1. PRISMA flowchart of the study.

\section{Results}

\section{Literature Search and Study Selection}

The search strategy identified 4,819 records. After duplicates removal, 2,757 studies were screened by title and abstract. Full texts of 82 articles were evaluated. Four others were not available. Ten publications met the inclusion criteria, with 3 duplicate publications. Finally, 7 studies were included, for a total of 401 patients [23-29]. The selection process is presented as a PRISMA flowchart (Fig. 1).

\section{Study Characteristics}

The baseline characteristics and methodological parameters of the included studies are summarized in Table 1. Three RCTs [23-25], two prospective [26, 27] and two retrospective $[28,29]$ cohort studies were included. Each study reported approval by an ethics committee when necessary.

Among the RCTs, the first had an IN placebo control group [23]. The second compared IN to IM sedation [24]. Milési et al. [25] study is a double-blind RCT with two IN interventions, both included in the outcome analysis.
One prospective study used a historical group as control [27]. None of the three other observational studies had a control group [26, 28, 29]. Only one study was multicentric [25]. All studies but one were in English. The study published in Chinese [24] was translated using a website (DeepL Translate, January 2021).

The indications for analgesia or sedation and the molecules used varied between studies. One study evaluated IN fentanyl for pain management during retinopathy of prematurity (ROP) screening [23]. Two studies described the sedative effect of IN midazolam or dexmedetomidine during MRI [24, 27]. IN midazolam (nMDZ) for intubation in the delivery room was evaluated in 2 studies [25, 26 , with comparison to IN ketamine (nKTM) in one [25]. One retrospective study documented the use of IN fentanyl for procedural pain management [28] and one focused on the safety and indications of IN fentanyl and midazolam within the NICU [29].

\section{Patient Characteristics}

In total, 401 patients were included: 327 preterms [23, 25-29] and 74 infants at term or term equivalent age [24, 


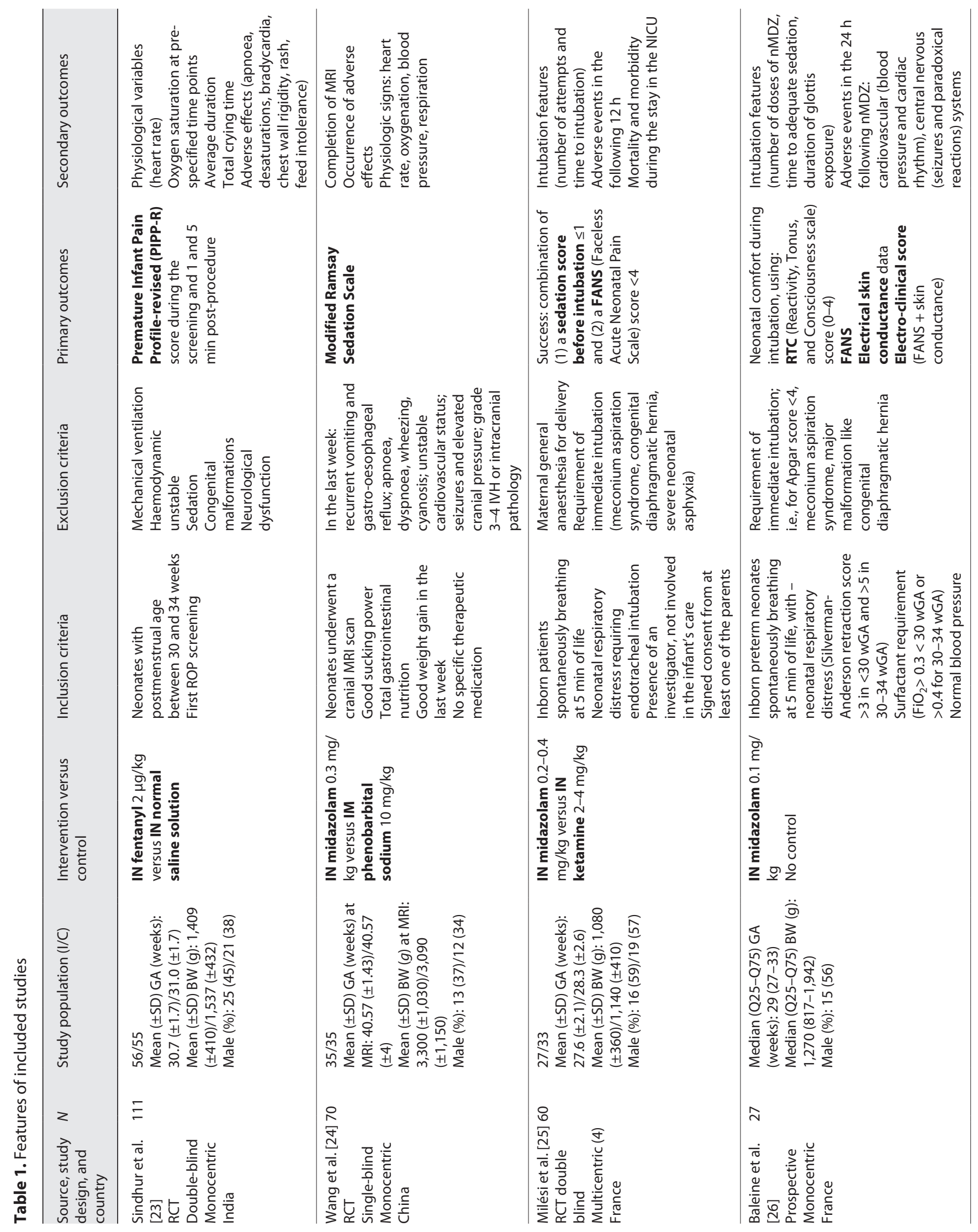




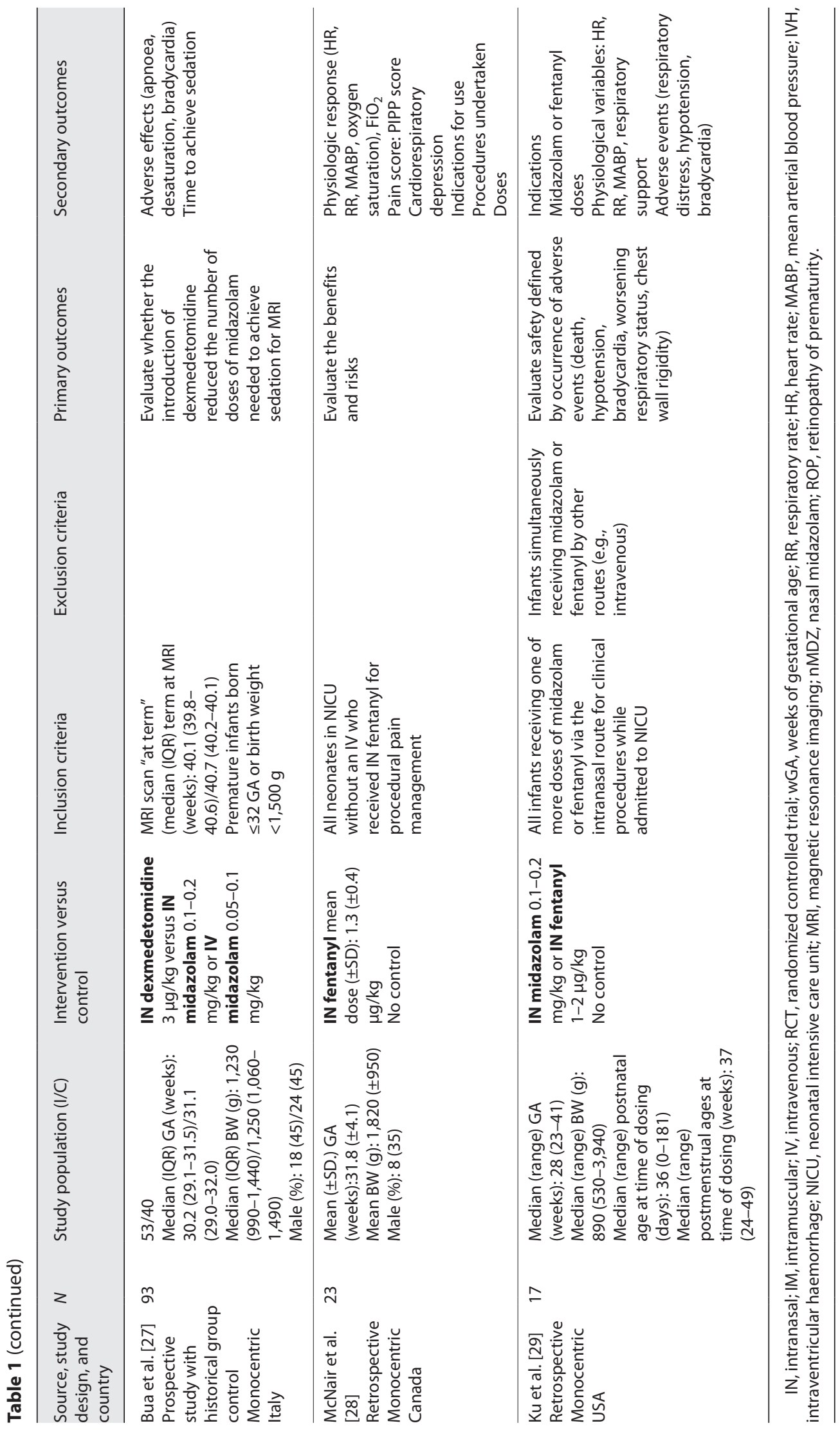


Fig. 2. Revised Cochrane risk-of-bias tool for (quasi)randomized trials (Rob2) and assessment of quality using the NewcastleOttawa Scale for cohort studies.

\begin{tabular}{|c|c|c|c|c|}
\hline Rob2 & $\begin{array}{l}\text { Sindhur } \\
(2020)\end{array}$ & $\begin{array}{l}\text { Wang } \\
(2020)\end{array}$ & $\begin{array}{l}\text { Milési } \\
(2020)\end{array}$ & \\
\hline Randomisation process & + & + & + & \\
\hline Deviations from the intended interventions & + & + & + & \\
\hline Missing outcome data & + & + & + & \\
\hline Measurement of the outcome & + & + & + & \\
\hline Selection of the reported result & + & + & + & \\
\hline \multirow[b]{2}{*}{ Quality assessment criteria } & Low & Low & Low & \\
\hline & $\begin{array}{l}\text { Baleine } \\
(2014)\end{array}$ & $\begin{array}{l}\text { Bua } \\
(2018)\end{array}$ & $\begin{array}{l}\text { McNair } \\
(2018)\end{array}$ & $\begin{array}{c}\mathrm{Ku} \\
(2019)\end{array}$ \\
\hline \multicolumn{5}{|l|}{ A. Selection } \\
\hline Representativeness of the exposed cohort & + & + & + & + \\
\hline Selection of the non-exposed cohort & - & + & - & - \\
\hline Ascertainment of exposure & + & - & + & + \\
\hline $\begin{array}{l}\text { Demonstration that outcome of interest was not present } \\
\text { at start of study }\end{array}$ & - & - & - & - \\
\hline \multicolumn{5}{|l|}{ B. Comparability } \\
\hline Study controls for gestational age/birthweight & I & + & 1 & / \\
\hline Study controls for at least 3 additional risk factors & I & + & I & I \\
\hline \multicolumn{5}{|l|}{ C. Outcome } \\
\hline Assessment of outcome & + & + & + & + \\
\hline Was follow-up long enough for outcome to occur? & + & + & + & + \\
\hline Adequacy of follow-up of cohorts & + & + & + & + \\
\hline Overall Quality Score (Maximum = 9) & 5 & 7 & 5 & 5 \\
\hline
\end{tabular}

$29]$. Five studies recruited only preterm infants [23, 2528]. Studies with a control group had well-matched gestational ages, birth weights, and sex ratio [23-25, 27].

\section{Risk of Bias}

The risk of bias of the three RCTs was assessed as low [23-25]. The quality of the cohort studies was limited by a lack of control group in three of them [26, 28, 29] and limitations in the methods description in the last [27]. Risks of bias and quality assessment criteria are presented in Figure 2.

\section{Outcome Analysis}

Main Outcome

The primary outcome was the assessment of pain and analgesia after IN drug administration in NICU patients, summarized in Figure 3. Five studies used standard pain and sedation scales to evaluate treatment effectiveness [23, 26-28]. Two studies assessing IN fentanyl used the Premature Infant Pain Profile (PIPP) [28] or its revision (PIPP-R) [23]. Sindhur et al. [23] reported a significant reduction in PIPP-R during ROP screening compared to IN saline solution $(p<0.001)$. PIPP scores indicative of adequate analgesia were also reported following IN use for various procedures [28].

Two studies evaluated both sedation and pain management using the Reactivity, Tonus, and Consciousness (RTC) scale and the Faceless Acute Neonatal pain Scale
(FANS) before and during intubation in the delivery room $[25,26]$. Comfort was rated as adequate in $85 \%$ of cases using pain thresholds of RTC $\leq 3$, FANS score $\leq 3$, and skin electrical conductance $\leq 0.21 \mathrm{pic} / \mathrm{s}$ [26]. Sedation was more frequently adequate after nMDZ than nKTM ( $93 \%$ vs. $64 \% ; p=0.04)$ while comfort was similar $(96 \%$ and $95 \%$ of patients, $p=1.00$ ) [25].

During MRI, IN midazolam improved the level of sedation as assessed by significantly higher modified Ramsay Sedation Scale scores $(p<0.05)$, compared with IM phenobarbital, $20 \mathrm{~min}$ after drug administration [24]. Another study of sedation for MRI reported that IN dexmedetomidine decreased the use of IV or IN midazolam. However, almost half of the infants (49\%) still required rescue therapy to complete the procedure [27].

Most studies analysed physiological parameters to consider pain assessment. A significant reduction in the heart rate after analgesia $[23,28]$ and significantly shorter crying times $(p=0.001)$ [23] were observed. No study described cerebral oxygenation by near-infrared spectroscopy.

\section{Secondary Outcomes}

Adverse events were reported in all studies, although with different incidences (Fig. 3). Five studies reported respiratory depression, apnoea, or desaturation, with similar incidences compared to control groups [23, 24, 27-29]. Respiratory depression was described as brief and self-limited or requiring only tactile stimulation [27]. 


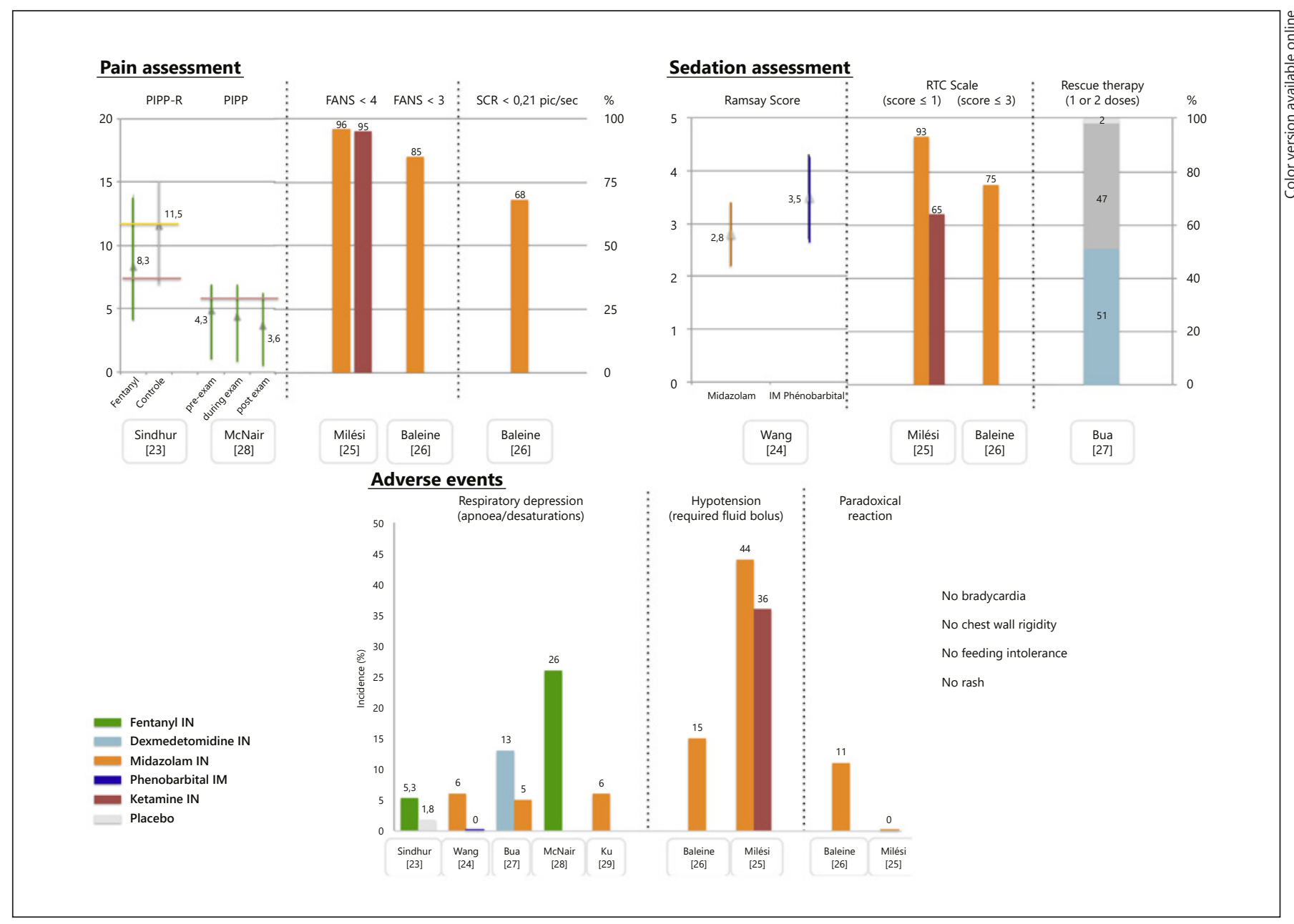

Fig. 3. Primary and secondary outcomes of the study: pain assessment; sedation assessment and adverses events. PIPP-R, Premature Infant Pain Profile-revised; PIPP, Premature Infant Pain Profile; FANS, Faceless Acute Neonatal pain Scale; SCR, skin conductance responses; RTC, Reactivity, Tonus and Consciousness; IM, intramuscular; IN intranasal.

Positive pressure ventilation or increased $\mathrm{FiO}_{2}[23,27$, 28 ] was only needed for short periods. Respiratory depression was mostly described after nMDZ $[24,27,29]$ or fentanyl administration [23, 28]. After dexmedetomidine, desaturation occurred in $13 \%$ of the infants, $85 \%$ of whom had been exposed to rescue therapy with IN or IV midazolam [27].

Baleine et al. [26] reported hypotension in 33\% of patients after midazolam. Fluid bolus for persistent hypotension was required in $15 \%$ and $44 \%$ of newborns following midazolam $[25,26]$ and in $36 \%$ after nasal ketamine [25]. These studies were conducted in preterm neonates in the delivery room. However, hypotension was not found after nasal midazolam $[24,29]$ or nasal fentanyl $[28,29]$ when used in the NICU.
One patient developed a paradoxical reaction, with agitation, myoclonus, and hypertension in the hour following a single nMDZ administration, and two other patients presented unsustained myoclonus immediately after administration [26]. Among other adverse events, bradycardia did not occur $[23,25-27,29]$, chest wall rigidity was not reported, particularly with fentanyl $[23,29]$, nor any rash or feeding intolerance [23].

One study described intermediate-term outcomes after $\mathrm{nMDZ}$ or $\mathrm{nKTM}$. There was no difference in mortality, morbidity, and NICU length of stay between the two groups [25].

IN drugs were most commonly used for analgesia or sedation during MRI $[24,27,29]$ or intubation [25, 26, 29]. Other indications included screening for ROP [23, 


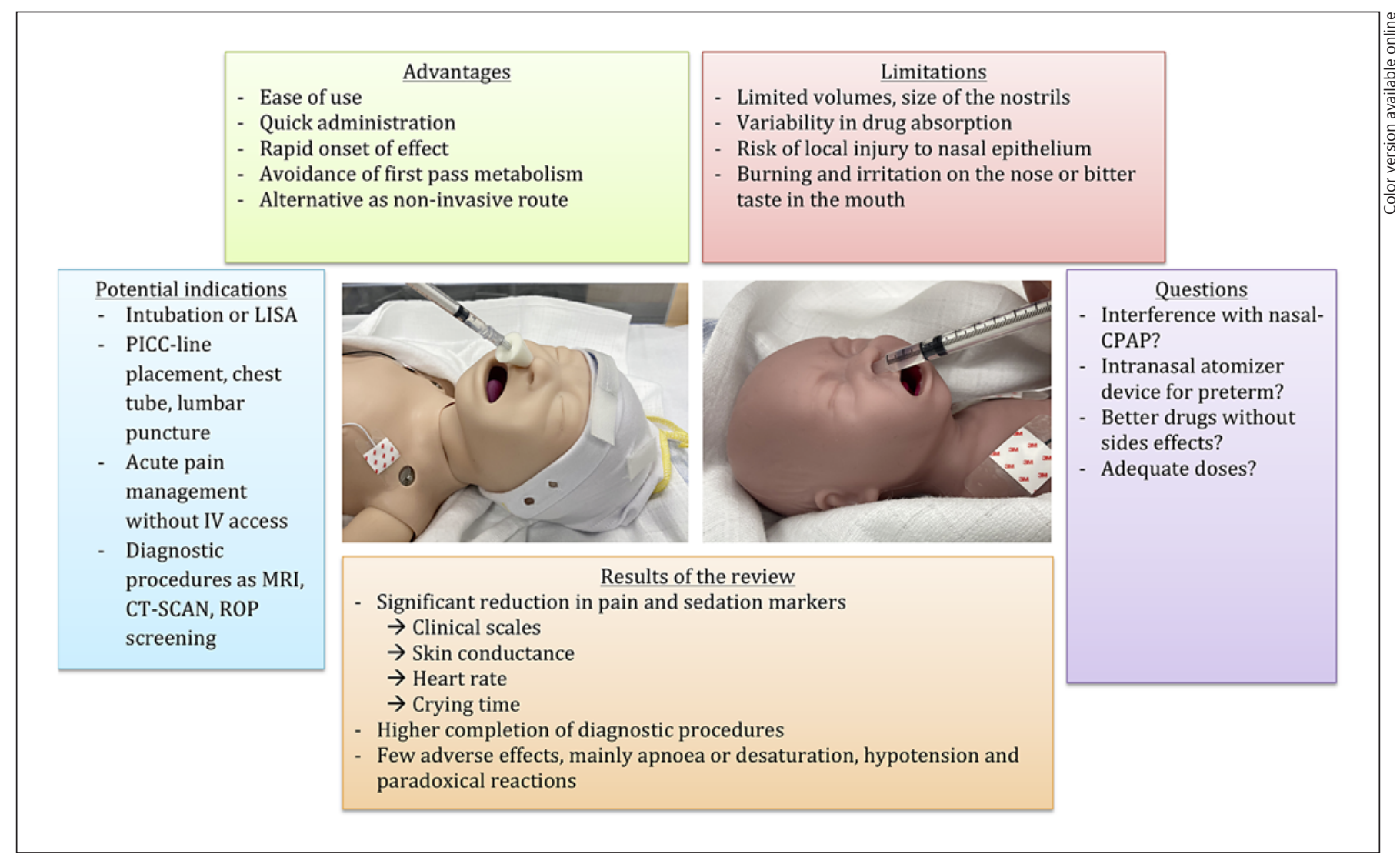

Fig. 4. Intranasal administration in neonates: summary. Pictures: left: IN administration with the atomizer, right: without it. IN, intranasal; CPAP, continuous positive airway pressure; LISA, less invasive surfactant administration; PICC, peripherally inserted central catheter; MRI, magnetic resonance imaging; CT-scan, computed tomography scan; ROP, retinopathy of prematurity.

29], central line insertion [28,29] or removal [28], thoracocentesis [28], and surgical dressing changes [29].

Molecules used included midazolam [24-27, 29], fentanyl [23, 28, 29], dexmedetomidine [27], and ketamine [25]. Doses of nasal midazolam ranged $0.1-0.4 \mathrm{mg} / \mathrm{kg}$, given once or twice $[24-27,29]$ with intervals before intervention at $4.8 \mathrm{~min}$ (IQR 3-9) and $10 \pm 6 \mathrm{~min}[25,26]$. Nasal ketamine with doses of $2-4 \mathrm{mg} / \mathrm{kg}$ allowed intubation after $16 \pm 8 \mathrm{~min}[25]$. IN fentanyl doses ranged from 1 to $2 \mu \mathrm{g} / \mathrm{kg}$ $[23,28,29]$. No additional pharmacokinetic data were available. A single dose of $3 \mu \mathrm{g} / \mathrm{kg}$ of IN dexmedetomidine achieved sedation within $10 \mathrm{~min}$ (IQR 8-12) [27].

\section{Discussion}

This systematic review identified 7 studies describing IN medications for pain and sedation management in NICU. The effectiveness of this alternative route to pro- vide analgesia was demonstrated by lower pain or sedation scores assessed by various scales, improvement of skin conductance responses (SCR), reduced crying times or heart rates variation during the procedure, higher completion of diagnostic exams such as MRI, or decreased use of rescue therapy. Side effects were reported infrequently and included statistically non-significant increases in apnoea or desaturation, hypotension, and paradoxical reactions, with agitation or myoclonus. IN sedation for delivery room intubation was associated with elevated incidence of hypotension requiring therapy. However, the lack of a placebo control group does not allow a comparison with the physiological hypotension reported in almost $20 \%$ of very preterm infants during neonatal adaptation [30]. The haemodynamic impact after midazolam or ketamine should be considered with caution. Moreover, reported complications were similar to those found after IV administration and more likely resulted from the molecule rather than its route of admin- 
Table 2. Intranasal drugs and characteristics in children and neonates

\begin{tabular}{|c|c|c|c|c|c|c|}
\hline \multirow[t]{3}{*}{ Midazolam } & \multirow[t]{3}{*}{$200-300$} & \multirow[t]{3}{*}{$50-200$} & \multirow[t]{3}{*}{$5-10$} & \multirow[t]{3}{*}{$4-6$} & \multirow[t]{3}{*}{$50-83$} & $\begin{array}{l}\text { Common: burning or irritation in nose, bitter taste in } \\
\text { mouth }\end{array}$ \\
\hline & & & & & & Uncommon: respiratory depression, hypotension \\
\hline & & & & & & $\begin{array}{l}\text { Rare: myoclonic activity and paradoxical agitation, } \\
\text { neurologic events }\end{array}$ \\
\hline \multirow[t]{3}{*}{ Fentanyl } & \multirow[t]{3}{*}{$1-2$} & \multirow[t]{3}{*}{$1-2$} & \multirow[t]{3}{*}{$2-5$} & \multirow[t]{3}{*}{1} & \multirow[t]{3}{*}{$55-70$} & $\begin{array}{l}\text { Common: local effects such as irritation and } \\
\text { discomfort }\end{array}$ \\
\hline & & & & & & Uncommon: nausea, vomiting \\
\hline & & & & & & Rare: bradycardia, chest wall rigidity \\
\hline \multirow[t]{2}{*}{ Ketamine } & \multirow[t]{2}{*}{$200-400$} & \multirow[t]{2}{*}{$100-300$} & \multirow[t]{2}{*}{$3-10$} & \multirow[t]{2}{*}{$1-3$} & \multirow[t]{2}{*}{$25-50$} & $\begin{array}{l}\text { Common/uncommon: spontaneously resolving } \\
\text { desaturation nausea, vomiting, cardiorespiratory } \\
\text { effects }\end{array}$ \\
\hline & & & & & & Rare: stridor, laryngospasm agitation \\
\hline
\end{tabular}

According to $[8,9,11,32,34,36,38,40,41]$.

istration. Data did not allow comparison for long-term outcomes in the sole prospective study where they were reported.

The IN route offers a possible alternative for drug delivery with a rapid onset action [31]. Its advantages and limitations, as well as its potential indications in neonates, are summarized in Figure 4. In children, IN administration appears to provide safe and efficient analgesia with pain control equivalent to oral or IV administration [8, $11,13,18]$. Its use is easy during either elective or urgent procedures especially when IV access is not available $[9$, 18]. A basic understanding of delivery techniques and medication bioavailability allows optimizing effectiveness [31]. The absorption of IN drugs is dependent on lipophilicity, molecular weight, drug ionization, and mucociliary clearance $[9,10]$. The highly vascularized nasal mucosa allows rapid absorption of drugs that bypass firstpass liver metabolism $[10,18]$. Maximizing both the drug concentration and the exposition to the absorptive mucosal surface increases absorption [18]. However, specific technical and safety questions about the IN route remain for the neonatal population. The use of atomizers is limited by the size of the neonatal nostril [18]. IN drugs are therefore administered without those, potentially decreasing mucosal exposition. While preterm infants often require non-invasive respiratory support, possible interference of nasal-CPAP or gas flows has not been investigated. As infants are obligatory nose breathers, potential local injury could lead to nasal obstruction. Burning or irritation in the nose and a bitter taste in the mouth have been reported in adults, especially after nMDZ [31, 32].

In addition to the paediatric and neonatal indications already mentioned, the extension of the use of IN in specific situations could be the subject of further reflection, including delivery room management of congenital malformations (e.g., diaphragmatic hernia and laparoschisis), less invasive surfactant administration, analgesia in severe dermatological diseases without IV access, or reduction of an incarcerated inguinal hernia. Few data are currently available for these potential indications.

Currently, the optimal choice of drugs and doses for analgesia in infants remains controversial [2]. Analgosedation protocols should take into account the pharmacokinetic characteristics of the medications (such as absorption rates and bioavailability), their pharmacodynamics according to gestational and postnatal ages with high inter-individual variability in preterm infants, and the respective depths of analgesia and sedation required for the procedure. A rapid onset of action is preferable for acute pain or short procedures such as intubation. For sedation 
such as during MRI, a more sustained effect is desired. Surveys reported that opioids, mainly morphine and fentanyl, are the most common treatments for pain in NICU, followed by midazolam, chloral hydrate, and phenobarbital. Alternative medications, such as ketamine, propofol, and dexmedetomidine, are less commonly used [2, 33]. The nasal doses used in the included studies were determined either by small population trials or by extrapolation from paediatric IN doses. Compared to IV doses, IN doses were broadly similar for fentanyl and slightly higher for the other molecules. Furthermore, pharmacokinetic data on bioavailability and absorption rate after IN administration are scarce in neonatology. Table 2 summarizes the paediatric and neonatal characteristics of the molecules used in the included studies and compares them with IV doses [34].

Fentanyl is an opioid considered to be 50-100 times more potent than morphine [35]. It has a rapid onset of action, from 2 to $5 \mathrm{~min}$, and a short half-life [7, 35]. In addition to inherent side effects of opioids such as respiratory depression, hypotension, constipation, and urinary retention, a risk of bradycardia and chest wall rigidity is recognized with fentanyl, typically with higher boluses and rapid injections [2,7]. Fentanyl is highly lipophilic, which increases its absorption after IN administration $[9,10]$ allowing good analgesia with few side effects $[23,27,28]$. In adults, its bioavailability is reported as between 55 and $70 \%$ of the equivalent IV dose [36]. Hence, McNair et al. [28] considered that 1.5 $\mu \mathrm{g} / \mathrm{kg}$ IN fentanyl is equivalent to $1 \mu \mathrm{g} / \mathrm{kg}$ IV. The interindividual variation of fentanyl pharmacokinetics is important in preterm infants [37]. As lower doses of 0.5 $\mu \mathrm{g} / \mathrm{kg}$ seemed to be insufficient for skin breaking procedures [37], doses of $1.5 \mu \mathrm{g} / \mathrm{kg} /$ dose (range from 1 to $2 \mu \mathrm{g} / \mathrm{kg} /$ dose) have been recommended for IN administration [35].

Midazolam is a short-acting benzodiazepine that acts through interaction with $\gamma$-aminobutyric acid receptors in the central nervous system, with sedative properties [38]. While midazolam remains widely used in routine practice in the NICU $[2,33]$, its use in preterm neonates is not recommended because of its side effects such as respiratory depression, hypotension, and long-term neurotoxicity $[2,25,39]$. Although additional safety data remain necessary, $\mathrm{nMDZ}$ at $0.1-0.4 \mathrm{mg} / \mathrm{kg}$ provided adequate sedation [24-27, 29]. IN bioavailability is around $50 \%$, and elimination half-life is between 4 and $12 \mathrm{~h}$ in the neonate $[32,38]$.

Among alternative molecules, dexmedetomidine, a selective alpha-2 adrenergic agonist, is increasingly used off label for paediatrics procedures and sedation within intensive care units [13-16]. It has sedative, anxiolytic, and analgesic properties and often reduces the need for concomitant opioid use [7]. The limited current neonatal data suggest limited efficacy in preterm and full-term infants, good clinical tolerance, absence of major side effects, and even a potential for neuroprotection [14]. Nasal dexmedetomidine has a high bioavailability $>80 \%$ [40], an onset of action of 15-30 min, and provides sedation for 55-100 $\min$ [41].

Ketamine is a dissociative anaesthetic that, in lower doses, provides good analgesia, amnesia, and sedation [2]. A recent meta-analysis concluded that IN ketamine produced adequate sedation in children, without serious adverse effects [12]. While nausea and vomiting were the most common side effects [12], occurrence of well-known neuropsychiatric effects, such as hallucinations or nightmares, was difficult to assess in neonates. Furthermore, its safety profile in neonates needs further characterization given possible neurotoxicity and limited data $[2,7]$. In infants, nKTM doses of $2-4 \mathrm{mg} / \mathrm{kg}$ provided adequate comfort during intubation [25]. The nKTM bioavailability of $25-50 \%$ is lower than those of nMDZ or nasal fentanyl [9].

This review's strengths include its systematic design, a pre-established protocol, and the application of the PRISMA methodology. The absence of language limitations improved exhaustivity.

The conclusions of this review will be limited, given the limited number of studies available and their small populations. Few presented a comparative arm either with a placebo or another route of administration, which limits our conclusions both on efficacy and safety. Data on pharmacokinetics and plasma levels are lacking and likely differ in term and preterm infants. Moreover, the inclusion of cohort studies increases the risk of publication bias.

Another difficulty highlighted with this review was the objective pain assessment in neonatology. The American Academy of Paediatrics suggested 5 scales to assess procedural neonatal pain, taking into account contextual and multidimensional factors, such as gestational age [2]. Those tools combine physiological and behavioural signs $[2,7]$. However, procedures can limit the evaluation of specific signs, such as facial responses with intubation. The FANS developed by Milési et al. [42] provided a specific solution in two of the studies $[25,26]$.

Changes in physiological variables have also been used for pain evaluation [2]. Baleine et al. [26] used electrical 
SCR, and most of the included studies evaluated the heart rate variability. Pain assessment in newborns remains challenging and requires the integration of multiple parameters.

The heterogeneity of outcomes is both a limitation and strength. The expected outcome of IN medications varied according to the procedure. While not allowing a metaanalysis and precise figures, this variability gave an overview of the possible uses of IN analgesia and sedation.

\section{Conclusion}

Despite limited data and heterogeneous studies, IN pain and sedation management tended toward a positive effect. Few general side effects such as brief apnoea/desaturation or hypotension were described. The IN route therefore appears to be a potential alternative for the administration of analgesic drugs in the NICU, particularly when IV access is not available. However, data addressing the safety of the IN medications and their pharmacokinetic are still insufficient in infants. There is a need for randomized control trials assessing, in both term and preterm infants, the effectiveness, safety, and pharmacology of IN medications for acute pain or for sedation, with comparisons with either IV medication or placebo when IV access is not possible.

\section{Statement of Ethics}

The research was conducted ethically in accordance with the World Medical Association Declaration of Helsinki. An ethics statement is not applicable because this study is based exclusively on the published literature. The protocol was registered in advance of data extraction with the Prospective Register of Systematic Reviews (registered on December 18, 2020; CRD42020221346).

\section{Conflict of Interest Statement}

The authors have no conflicts of interest to declare.

\section{Funding Sources}

There was no financial support for the review.

\section{Author Contributions}

D.S. and S.T. contributed to the search strategy, data selection, and analysis. D.S. drafted and revised the manuscript. V.R. helped with data interpretation, writing, and editing of the manuscript. All authors reviewed and approved the manuscript.

\section{Data Availability Statement}

All data analysed are included in this article and its online supplementary material files. Further enquiries can be directed to the corresponding author.

\section{References}

1 Carbajal R, Rousset A, Danan C, Coquery S, Nolent P, Ducrocq S, et al. Epidemiology and treatment of painful procedures in neonates in intensive care units. JAMA. 2008;300(1): 60-70.

2 Statement P. Prevention and management of procedural pain in the neonate: an update. Pediatrics. 2016;137(2):e20154271.

3 Anand KJS, Aynsley-Green A, Bancalari E, Benini F, Champion GD, Craig KD, et al. Consensus statement for the prevention and management of pain in the newborn. Arch Pediatr Adolesc Med. 2001;155(2):173-80.

4 Anand KJ, Hickey PR. Pain and its effects on the human neonate and fetus. N Engl J Med. 1987 Nov 19;317(21):1321-9.

5 Brummelte S, Grunau RE, Chau V, Poskitt KJ, Brant R, Vinall J, et al. Procedural pain and brain development in premature newborns. Ann Neurol. 2012;71(3):385-96.

6 Vinall J, Miller SP, Chau V, Brummelte S, Synnes AR, Grunau RE. Neonatal pain in relation to postnatal growth in infants born very preterm. Pain. 2012;153(7):1374-81.

7 Carter BS, Brunkhorst J. Neonatal pain management. Semin Perinatol. 2017;41(2):111-6.
8 Mudd S. Intranasal fentanyl for pain management in children: a systematic review of the literature. J Pediatr Health Care. 2011;25(5): 316-22.

9 Setlur A, Friedland H. Treatment of pain with intranasal fentanyl in pediatric patients in an acute care setting: a systematic review. Pain Manag. 2018;8(5):341-52.

10 Devillier P, Grassin-Delyle S, Naline E, Buenestado A, Blouquit-Laye S, Advenier C. Administration intranasale des médicaments à visée systémique: Une nouvelle voie pour les opioïdes. Therapie. 2010;65(5):475-81.

11 Mittal P, Manohar R, Rawat AK. Comparative Study of intranasal midazolam and intravenous diazepam sedation for procedures and seizures. Indian J Pediatr. 2006;73(11):975-8.

12 Poonai N, Canton K, Ali S, Hendrikx S, Shah A, Miller M, et al. Intranasal ketamine for anesthetic premedication in children: a systematic review. Pain Manag. 2018;8(6):495-503.

13 Li L, Zhou J, Yu D, Hao X, Xie Y, Zhu T. Intranasal dexmedetomidine versus oral chloral hydrate for diagnostic procedures sedation in infants and toddlers: a systematic review and meta-analysis. Medicine. 2020;99(9):e19001-7.
14 Baier NM, Mendez SS, Kimm D, Velazquez $\mathrm{AE}$, Schroeder AR. Intranasal dexmedetomidine: an effective sedative agent for electroencephalogram and auditory brain response testing. Paediatr Anaesth. 2016;26(3):280-5.

15 Liu H, Sun M, Zhang J, Tian Q, Yu Q, Liu Y, et al. Determination of the $90 \%$ effective dose of intranasal dexmedetomidine for sedation during electroencephalography in children. Acta Anaesthesiol Scand. 2019;63(7):847-52.

16 Li BL, Yuen VM, Song XR, Ye J, Ni J, Huang $\mathrm{JX}$, et al. Intranasal dexmedetomidine following failed chloral hydrate sedation in children. Anaesthesia. 2014;69(3):240-4.

17 Harlos MS, Stenekes S, Lambert D, Hohl C, Chochinov HM. Intranasal fentanyl in the palliative care of newborns and infants. J Pain Symptom Manage. 2013;46(2):265-74.

18 Kaushal S, Placencia JL, Maffei SR, Chumpitazi CE. Intranasal fentanyl Use in Neonates. Hosp Pharm. 2020;55(2):126-9.

19 Page MJ, McKenzie JE, Bossuyt PM, Boutron I, Hoffmann TC, Mulrow CD, et al. The PRISMA 2020 statement: An updated guideline for reporting systematic reviews. BMJ. 2021:372; n71. 
20 Ouzzani M, Hammady H, Fedorowicz Z, Elmagarmid A. Rayyan-a web and mobile app for systematic reviews. Syst Rev. 2016;5(1): 210.

21 Higgins JPT, Altman DG, Gøtzsche PC, Jüni $\mathrm{P}$, Moher D, Oxman AD, et al. The cochrane collaboration's tool for assessing risk of bias in randomised trials. BMJ. 2011;343(7829): $1-9$.

22 Wells GA, Shea B, O'Connell D, Peterson J, Welch V, Losos MPT. The Newcastle-Ottawa Scale (NOS) for assessing the quality of nonrandomised studies in meta-analyses [Internet]. Available from: http://www.ohri.ca/programs/clinical_epidemiology/oxford.asp\#. YMZeXAIl_Q4.gmail.

23 Sindhur M, Balasubramanian $\mathrm{H}$, Srinivasan L, Kabra NS, Agashe P, Doshi A. Intranasal fentanyl for pain management during screening for retinopathy of prematurity in preterm infants: a randomized controlled trial. J Perinatol. 2020;40(6):881-7.

24 Wang FH, Zhang J, Xiao M, Wu F, Liu L, Zhou XH. Sedative effect of intranasal midazolam in neonates undergoing magnetic resonance imaging: a Prospective Single-Blind Randomized Controlled Study. Chinese J Contemp Pediatr. 2020;22(5):441-5.

25 Milési C, Baleine J, Mura T, Benito-Castro F, Ferragu F, Thiriez G, et al. Nasal midazolam versus ketamine for neonatal intubation in the delivery room: a randomised trial. Arch Dis Child Fetal Neonatal Ed. 2018;103(3): F221-6.

26 Baleine J, Milési C, Mesnage R, Novais ARB, Combes C, Durand S, et al. Intubation in the delivery room: Experience with nasal midazolam. Early Hum Dev. 2014;90(1):39-43.
27 Bua J, Massaro M, Cossovel F, Monasta L, Brovedani P, Cozzi G, et al. Intranasal dexmedetomidine, as midazolam-sparing drug, for MRI in preterm neonates. Paediatr Anaesth. 2018;28(8):747-8.

28 McNair C, Graydon B, Taddio A. A Cohort Study of intranasal fentanyl for procedural pain management in neonates. Paediatr Child Health. 2018;23(8):e170-5.

29 Ku LC, Simmons C, Smith PB, Greenberg RG, Fisher K, Hornik CD, et al. Intranasal midazolam and fentanyl for procedural sedation and analgesia in infants in the neonatal intensive care unit. J Neonatal Perinatal Med. 2019; 12(2):143-8.

30 Dempsey EM, Barrington KJ, Marlow N, O’Donnell CPF, Miletin J, Naulaers G, et al. Hypotension in preterm infants (HIP) randomised trial. Arch Dis Child Fetal Neonatal Ed. 2021;106(4):398-403.

31 Wolfe TR, Braude DA. Intranasal medication delivery for children: a brief review and update. Pediatrics. 2010;126(3):532-7.

32 Pacifici GM. Clinical pharmacology of midazolam in neonates and children: effect of disease-a review. Int J Pediatr. 2014;2014: 309342.

33 Carbajal R, Eriksson M, Courtois E, Boyle E, Avila-Alvarez A, Andersen RD, et al. Sedation and analgesia practices in neonatal intensive care units (EUROPAIN): results from a Prospective Cohort Study. Lancet Respir Med. 2015;3(10):796-812.

34 Tesoro S, Marchesini V, Fratini G, Engelhardt T, De Robertis E. Drugs for anesthesia and analgesia in the preterm infant. Minerva Anestesiol. 2020;86(7):742-55.
35 Thigpen JC, Odle BL, Harirforoosh S. Opioids: a review of pharmacokinetics and pharmacodynamics in neonates, infants, and children. Eur J Drug Metab Pharmacokinet. 2019; 44(5):591-609.

36 Lim SC, Paech MJ, Sunderland VB, Roberts MJ, Banks SL, Rucklidge MW. Pharmacokinetics of nasal fentanyl. J Pharm Pract Res. 2003;33(1):59-64.

37 Norman E, Kindblom JM, Rane A, Berg AC, Schubert U, Hallberg B, et al. Individual variations in fentanyl pharmacokinetics and pharmacodynamics in preterm infants. Acta Paediatr Int J Paediatr. 2019;108(8):1441-6.

38 Allegaert K. The clinical pharmacology of short acting analgo-sedatives in neonates. Curr Clin Pharmacol. 2011;6(4):222-6.

$39 \mathrm{Ng}$ E, Taddio A, Ohlsson A. Intravenous midazolam infusion for sedation of infants in the neonatal intensive care unit. Cochrane Database Syst Rev. 2017;1(1):CD002052.

40 Miller JW, Balyan R, Dong M, Mahmoud M, Lam JE, Pratap JN, et al. Does intranasal dexmedetomidine provide adequate plasma concentrations for sedation in children: a Pharmacokinetic Study. Br J Anaesth. 2018;120(5): 1056-65.

41 Mondardini MC, Amigoni A, Cortellazzi P, Di Palma A, Navarra C, Picardo SG, et al. Intranasal dexmedetomidine in pediatrics: update of current knowledge. Minerva Anestesiol. 2019;85(12):1334-45.

42 Milesi C, Cambonie G, Jacquot A, Barbotte E, Mesnage R, Masson F, et al. Validation of a neonatal pain scale adapted to the new practices in caring for preterm newborns. Arch Dis Child Fetal Neonatal Ed. 2010;95(4):263- 